\title{
Regulatory effects of GRK2 on GPCRs and non-GPCRs and possible use as a drug target (Review)
}

\author{
CHEN-CHEN HAN, YANG MA, YIFAN LI, YANG WANG and WEI WEI \\ Institute of Clinical Pharmacology, Anhui Medical University, Key Laboratory of Anti-inflammatory \\ and Immune Medicine, Ministry of Education, Anhui Collaborative Innovation Center of \\ Anti-inflammatory and Immune Medicine, Hefei, Anhui 230032, P.R. China
}

Received May 5, 2016; Accepted August 22, 2016

DOI: $10.3892 /$ ijmm.2016.2720

\begin{abstract}
G protein-coupled receptor kinase 2 (GRK2) is a key member of the $G$ protein-coupled receptor kinase (GRK) family. GRK2 activity is regulated by the C-terminus of GRK2 which contains a plekstrin homology domain and the N-terminus of GRK2 which contains the RGS homology domain with binding sites for several proteins and lipids such as $G$ protein-coupled receptors (GPCRs), G protein, phospholipase C, phosphatidylinositol 4,5-bisphosphate, extracellular signal-regulated kinase, protein kinase A and G $\beta \gamma$. GRK2 phosphorylates the GPCR and enhances the affinity of binding to arrestins, which uncouple the receptors from $\mathrm{G}$ proteins, and target the receptors for desensitization and internalization. GRK2 also regulates non-GPCR desensitization and internalization by phosphorylation, and is important in maintaining the balance between the receptors and signal transduction. Previous findings have indicated that the upregulation of GRK2 in heart failure enhances dysfunctional adrenergic signaling and myocyte death. Collagen-induced arthritis induces the upregulation of GRK2 expression in fibroblast-like synoviocytes. In this review, we discussed the evidence for the association between altered GRK2 levels and various diseases, which suggests that GRK2 may be an effective drug target for preventing and treating heart failure, hypertension and inflammatory disease.
\end{abstract}

\section{Contents}

1. Introduction

2. Structural features of GRK2

3. Modulation of GRK2 activity

Correspondence to: Professor Wei Wei or Dr Yang Ma, Institute of Clinical Pharmacology, Anhui Medical University, Key Laboratory of Anti-inflammatory and Immune Medicine, Ministry of Education, 81 Meishan Road, Hefei, Anhui 230032, P.R. China

E-mail: wwei@ahmu.edu.cn

E-mail: mayang_ahmu@126.com

Key words: G protein-coupled receptor kinase 2, G protein-coupled receptor, signal transduction, drug targets, disease
4. Effects of GRK2 on receptor function

5. Possible effects of GRK2 in some diseases

6. Conclusion

\section{Introduction}

G protein-coupled receptor kinase 2 (GRK2) belongs to a unique family of Ser/Thr protein kinases which are best known for their role in the rapid desensitization of $G$ protein-coupled receptors (GPCRs) (1). The structural architecture of GRK2 comprises a central catalytic domain, an N-terminal domain which contains an $\mathrm{RH}$ domain (regulator of $\mathrm{G}$ protein signaling (RGS) homology domain) and a C-terminal domain. These domains have been proposed to be important in regulating signal transduction and membrane positioning function. GRK2 activity is regulated by interactions with protein and lipid, including among others extracellular signal-regulated kinase (ERK), protein kinase A (PKA), protein kinase $C(P K C)$ and $G \beta \gamma(2)$. Furthermore, GRK2 is also one of the key participants in signal transduction mediated through GPCRs as well as in the internalization and resensitization of GPCRs. GRK2 is broadly distributed and participates in the regulation of $\beta$-adrenergic receptor $(\beta-A R)$, angiotensin $\mathrm{II}_{1 \mathrm{~A}}$ receptor $\left(\mathrm{AT}_{1 \mathrm{~A}}-\mathrm{R}\right)$ and chemokine receptors amongst others, which play important roles in vascular function, immunity and inflammation.

The protein and mRNA levels of GRK2 in peripheral blood lymphocytes are increased in patients with heart failure (HF) or hypertension, and collagen-induced arthritis (CIA) induces the upregulation of GRK2 expression in fibroblast-like synoviocytes (FLSs). GRK2 is upregulated in heart failure, hypertension, CIA and cancer, and it appears to play a critical role in disease progression $(38,43)$. Previous research has shown that the inhibition of cardiac GRK2 improves the functional and morphological parameters of the failing heart and reduces cardiolipotoxicity by promoting a cardiomyocyte survival program (3). Decreased GRK2 expression may promote adrenergic receptor (AR)-stimulated vasodilation and protect against angiotensin II (Ang II)-induced hypertension by increasing nitric oxide bioavailability (4). Thus, the inhibition of GRK2 may represent a viable therapeutic option for the treatment of diseases including HF, hypertension and CIA. 


\section{Structural features of GRK2}

The GRK2 tertiary structure domain contains a central catalytic domain, an $\mathrm{N}$-terminal domain and a carboxyl-terminal domain (Fig. 1). The N-terminus of GRK2 contains the RH domain which plays a vital role in receptor recognition and intracellular membrane anchoring. The C-terminal domain of GRK2 may promote subcellular localization and agonist-dependent translocation by combining with lipids and other membrane proteins. The N-terminus of GRK2 interacts selectively with Gaq, and inhibits the Gaq-mediated activation of phospholipase C (PLC) by sequestering G $\alpha$. The C-terminus plekstrin homology (PH) domain of GRK2, which interacts with phosphatidylinositol 4,5-bisphosphate (PIP2) and free $G \beta \gamma$ subunits, can mediate the agonist-dependent translocation of GRK2 to the plasma membrane.

\section{Modulation of GRK2 activity}

GRK2 exists on the cell membrane and microsome membrane. It was originally described as a soluble, cytosolic enzyme that transiently translocates to the plasma membrane upon receptor activation. However, GRK2 activity is regulated by interactions with protein and lipid. The ERK phosphorylation site at Ser670 and the S-nitrosothiols (SNOs) S-nitrosylation site at Ser340 lead to the inhibition of GRK2; the PKA phosphorylation site at Ser685, Tyr-13, 86 and 92 of GRK2 phosphorylation and $\mathrm{G} \beta \gamma$ binding can promote GRK2 activation.

Activation of ERK inhibits GRK2 activity. GRK2 plays an important role in the stimulation of the ERK/mitogen-activated protein kinase (MAPK) cascade to regulate the function of $\beta_{2}$-AR. The inhibition of ERK activity potentiates GRK2 activity, and the activation of $\beta_{2}$-AR promotes the phosphorylation of GRK2 regulated by ERK (5). COS-7 cells were transfected with GRK2, active MEK1, dominant-negative (DN) MEK and $\beta_{2}$-AR and treated with the $\beta$-agonist isoproterenol for different periods of time. DN MEK strongly promoted a marked (2-3-fold) increase in the phosphorylation of $\beta_{2}$-AR by GRK2, and active MEK1 weakly inhibited the phosphorylation of $\beta_{2}$-AR by GRK2. Thus, active MEK was considered as a kinase to promote GRK2 phosphorylation; it inhibited GRK2 kinase activity leading to the increased phosphorylation of GRK2, which finally inhibited GRK2 activity and the activation of $\beta_{2}$-AR by GRK2. Conversely, GRK2 phosphorylation by DN MEK1 results in the promotion of $\beta_{2}$-AR phosphorylation by GRK2. The phosphorylation of GRK2 by ERK at Ser670 may inhibit GRK2 kinase activity, and this plays an important role in regulating the function of the receptor.

SNOs decrease GRK2 activity. Previous research has shown that S-nitrosylation of GRK2 at Cys 340 by CysNO leads to the inhibition of GRK2 activity and the decreased phosphorylation of $\beta_{2}$-AR (6). To identify S-nitrosylation sites in GRK2, experiments were performed by mapping the sites of S-nitrosylation among the 15 cysteines of GRK2. 293 cells were transfected with Y326A $\beta_{2}$-AR, GRK2 wild-type (wt), GRK2 C340S and GRK2 C439S. CysNO inhibited $\beta_{2}$-AR phosphorylation by either GRK2 wt or C439S but had no effect on receptor phosphorylation by GRK2 C340S. The results suggested that GRK2 is S-nitrosylated at the site of Cys340. S-nitrosylation inhibits exogenous GRK2 and endogenous GRK2 phosphorylation as well as S-nitrosylation. In addition, S-nitrosylation of GRK2 is associated with $\beta_{2}$-AR internalization; S-nitrosylation of GRK2 showed a significant decrease in agonist-stimulated $\beta_{2}$-AR internalization, whereas cells expressing GRK2 C340S actually showed a slight increase in agonist stimulated $\beta_{2}$-AR internalization.

Binding of $G \beta \gamma$ promotes GRK2 activity. Free G $\beta \gamma$ subunits bind to GRK2 with high affinity and are involved in the association of GRK2 to lipid vesicles and GPCR phosphorylation in reconstituted systems (7). Binding of $G \beta \gamma$ promotes GRK2 activity upon enhancing the GPCR-mediated allosteric activation of GRK2. GRK2 has multisite contact with G $\beta \gamma$ involving the PH domain Arg-587 and Lys-663, 665 and 667. Notably, the Arg-587 mutant not only reduces the interaction of GRK2 with G $\beta \gamma$ but also impairs the allosteric activation of GRK2, which suggests that Arg-587 may be an important site for inducing the conformational switch of GRK2. In addition, G $\beta \gamma$ subunits aid in the targeting of GRK2 to membranes and may also assist in determining the substrate specificity of these enzymes. The $\mathrm{N}$-terminus of GRK2 (1-55aa) was also a G $\beta \gamma$-binding site.

PKA-mediated GRK2 phosphorylation. Agonist-stimulated PKA-mediated phosphorylation of GRK2 may represent a mechanism for enhancing receptor phosphorylation and desensitization (8). GRK2 has three potential sites of PKA phosphorylation, including S670, S676 and S685. To investigate PKA phosphorylation sites on GRK2, high pressure liquid chromatography fractionation and sequencing of radiolabeled peptides results showed that the residues $678-689$ of GRK2 contain a single site of phosphorylation at serine 685 . Thus, PKA directly phosphorylates GRK2 on serine 685 . PKA activated by Gs-coupled receptors phosphorylates GRK2, enhances G $\beta \gamma$ subunit binding to membrane targeting and enhances the ability of the kinase to translocate to the membrane and phosphorylate the receptor. Mutation of S685 to alanine (S685A) greatly attenuated GRK2-mediated translocation to $\beta_{2}$-AR and the phosphorylation of agonist-occupied $\beta_{2}$-AR leading to a subsequent decrease in receptor internalization.

\section{Effects of GRK2 on receptor function}

As the most widely expressed member of GRK family, GRK2 plays an important role in the regulation of GPCRs and non-GPCRs (Table I). GRK2 phosphorylates GPCRs and enhances the affinity of binding to arrestins, which uncouple the receptors from $G$ proteins, and target the receptors for desensitization and internalization (Fig. 2A). G protein is not involved in the phosphorylation of GRK2 on non-GPCRs, and the association of $\beta$-arrestin1 ( $\beta$-arr1) and insulin-like growth factor-1 receptor (IGF-1R) is one of transient binding (Fig. 2B).

Regulatory effects of GRK2 on $\beta_{2}-A R$. $\beta_{2}$-AR is a GPCR with a common structural signature of seven membrane-spanning helices (3). $\beta_{2}$-AR which is activated by epinephrine plays a key role in physiologic cardiovascular processes. The $\beta_{2}$-AR contains relatively unstructured regions that are involved in functionally important protein-protein interactions. The C-terminus and 


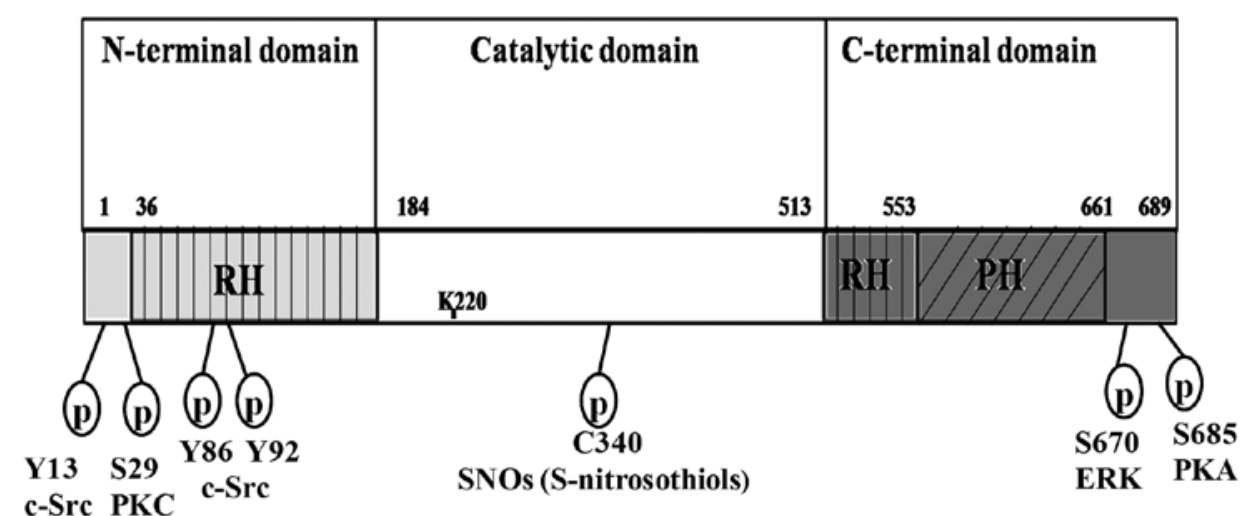

Figure 1. G protein-coupled receptor kinase 2 (GRK2) structural domains and phosphorylation sites. GRK2 has an N-terminal region (light grey), which is implicated in G protein-coupled receptor (GPCR) binding, followed by RGS homology (RH) domain (vertical stripes). The RH domain is interrupted by the catalytic domain shared by all kinases (white). GRK2 also has a C-terminal pleckstrin homology (PH) domain (diagonal stripes) implicated in binding anionic phospholipids and G $\beta \gamma$. The position of the key lysine is responsible for catalysis in the kinase domain. The mutations in K220R in GRK2 yield kinase-dead GRK2; a c-Src site at Tyr13, 86 and 92, a protein kinase C (PKC) site at Ser29, an extracellular signal-regulated kinase (ERK) site at Ser670, and a protein kinase A (PKA) site at Ser685; GRK2 was S-nitrosylated by NO with the primary site Cys340.

the third intracellular loop of $\beta_{2}$-AR are the most unstructured regions (9). The $\mathrm{N}$ - and $\mathrm{C}$-terminal ends of the third intracellular loop of $\beta_{2}$-AR are associated with $G$ protein activation and the selectivity of the interaction of GPCR and G protein. The C-terminus of $\beta_{2}$-AR interacts with GRK2, arrestins and other signaling molecules (10). In 293 cells, the phosphorylation of $\beta_{2}$-AR is regulated by GRK2, which promotes $\beta_{2}$-AR desensitization and internalization. To identify the phosphorylation sites on the $\beta_{2}$-AR for which GRK2 is responsible, experiments were performed involving the silencing of these kinases separately from cells and subsequently using stable isotope labeling with amino acids in cell culture (SILAC) to quantitatively measure the extent of phosphorylation at each site in cells stimulated with isoproterenol. The following GRK2 phosphorylation sites of $\beta_{2}$-AR were examined: Thr360, Ser364, Ser396, Ser401, Ser407 and Ser411 (11).

Regulatory effects of GRK2 on angiotensin II receptor. The $\mathrm{AT}_{1 \mathrm{~A}}-\mathrm{R}$ is well known to mediate multifarious angiotensin-dependent physiological responses such as vasoconstriction, smooth muscle cell motility and growth, and secretion (12). Furthermore, Ang II type-1 (AT1) receptors exert complex and diverse physiological actions which are associated with a number of diseases or disorders such as hypertension, hypertrophy, fibrosis, thrombosis and atherosclerosis. It demonstrates that overexpression of GRK2 significantly promotes agonist-induced phosphorylation of $\mathrm{AT}_{1 \mathrm{~A}}-\mathrm{R}$ in 293 cells. Knockdown of GRK2 leads to a significant decrease in the internalization of the $\mathrm{AT}_{1 \mathrm{~A}}-\mathrm{R}$, and it mainly slows down the speed of $\mathrm{AT}_{1 \mathrm{~A}}-\mathrm{R}$ internalization (13). It has been suggested that GRK2 may promote $\mathrm{AT}_{1 \mathrm{~A}} \mathrm{R}$ overexpression in the myocardium, which attenuated contractility and heart rate in response to Ang II (14). GRK2 as a major kinase may promote the phosphorylation of $\mathrm{AT}_{1 \mathrm{~A}}-\mathrm{R}$, and it can regulate $\beta$-arrestin-mediated internalization and endocytosis of $A T_{1 A}-R$.

Regulatory effects of GRK2 on chemokine receptor. Chemotactic chemokine, relative molecular mass of 7-10 kDa of proinflammatory cytokines, can be produced by a variety of cells. Chemokine receptors are widely expressed in various cells of the immune system and regulate the immune cells during the inflammatory response. The activation of white blood cells and the chemotaxis function of chemokines play an important role in a number of physiological and pathological processes such as embryonic development, angiogenesis and tumor development. The activation of $\mathrm{T}$ cells produces different chemokines and their receptors; these substances are capable of inducing a target cell to migrate to the inflammatory site.

Many studies have shown the involvement of GRK2 in the desensitization and internalization of chemokine receptors such as CCR5, CCR2, CXCR4, CXCR2 as well as chemotactic receptors for substance P. GRK2 expression and kinase activity are associated with ERK1/2 and MEK1/2 of chemokine receptor signaling in astrocytes (15). A decreased GRK2 level significantly promoted the CCL2-induced phosphorylation of ERK1/2. Overexpression of GRK2 inhibited CCL2-induced Akt phosphorylation without altering CCL2-induced ERK1/2 phosphorylation. Simultaneously, chemokine-induced Akt phosphorylation required the kinase activity of GRK2, but the change in the activation of GRK2 was not sufficient to alter CCL2-induced ERK1/2 phosphorylation (15). In astrocytes, GRK2 controls the internalization of CXCR7 and regulates SDF-1/CXCL12 of CXCR7-mediated signaling (16). Chemokine receptor and the related substances regulated by GRK2 are associated with the migration of white blood cells to the inflammatory lesion as well as the activation and proliferation of white blood cells.

Regulatory effects of $G R K 2$ on IGF-1R. IGF-1R is a transmembrane receptor tyrosine kinase (RTK), and it regulates cellular proliferation, survival and metastasis, making this receptor an attractive target for cancer treatment (28). A previous study showed that GRK2 as serine kinases for IGF-1R, DN GRK2 mutant resulted in reduced cellular proliferation by regulating the IGF-1R (29). The GRK2-dependent phosphorylation of IGF-1R serine residues is the underlying mechanism responsible for $\beta$-arrestin binding to these residues, and serines 1248 and 1291 are the major phosphorylation sites of the IGF-1R. In 293T cells, GRK2 overexpression enhanced the association of GRK2 with the IGF-1R, augmented IGF-1-induced ubiquitination, and increased basal levels of $\beta$-arr1/IGF-1R association. 
Table I. GRK2 regulates GPCRs and non-GPCRs.

Type of receptor

Function

Refs.

\section{GPCRs}

$\beta_{2}$-adrenergic receptor $\left(\beta_{2}\right.$-AR)

Angiotensin II receptor (ATII-R)

Chemokine receptor

Prostaglandin receptor (EP-R)

Adenosine receptors

$\mathrm{TP} \beta$ isoform of human

thromboxane $\mathrm{A}_{2}$ receptor (TP)

GABAB receptors (GABABR)

Lysophosphatidic acid

receptor (LPAr)

Dopamine receptor

Smo

Non-GPCRs

Platelet-derived growth factor receptor- $\beta$ (PDGFR)

Insulin-like growth factor-1

receptor (IGF-1R)

Epidermal growth factor receptor (EGFR)
GRK2-mediated phosphorylation promotes

desensitization and internalization of $\beta_{2}$-AR

GRK2 promotes phosphorylation of $\mathrm{AT}_{1 \mathrm{~A}} \mathrm{R}$, and

participates in $\beta$-arrestin-mediated internalization

and endocytosis of $\mathrm{AT}_{1 \mathrm{~A}}$

GRK2 involved in the desensitization and

internalization of chemokine receptors

Overexpression of GRK2 augmented agonist-induced,

FLAG-rEP3hEP4-Ct-R phosphorylation

GRK2-mediated desensitization of adiponectin receptor

Effect of overexpressing a dominant-negative form

of GRK2 on agonist-induced desensitization of TP $\beta$

Co-expression of GRK2 had no effect on GABA(B)

receptor-mediated desensitization processes

GRK2 and $\beta$-arrestin1 desensitized LPAr-mediated signaling

and regulated LPA-stimulated functional effects in FRTL-5 cells

GRK2 was capable of regulating D2DAR activity in

the absence of receptor phosphorylation

GRK2-mediated phosphorylation of Smo signaling cascade

GRK2-mediated phosphorylation induced desensitization of PDGFR $\beta$

GRK2 negatively regulated IGF-1R signaling pathway

GRK2 promoted serine phosphorylation of EGFR

GRK2 interacts with GPCRs and non-GPCRs, and participates in the regulation of GPCR and non-GPCR signaling pathways. GRK2, G protein-coupled receptor kinase 2; GPCRs, G protein-coupled receptors.

\section{Possible effects of GRK2 in some diseases}

GPCRs are involved in regulating the actions of messengers such as catecholamines, endothelins, angiotensins and chemokines. Such substances are important regulators of cardiovascular functions, which participate in cardiovascular development, and in controlling the growth and remodeling of myocardial cells. Simultaneously, the desensitization of GPCR contributes to the regulation of cardiovascular disease. The $\beta$-adrenergic receptor, Ang II AT1R and chemokine receptor are regulated by GRK2; these receptors are known to play a central role and represent an important target for the treatment of chronic HF, angina pectoris and hypertension, rheumatoid arthritis and malignant tumors. GRK2 inhibition may prove to be positive and valuable as a therapy for these diseases.

Potential role of GRK2 in HF. HF is a chronic clinical syndrome, which is characterized by reduced left ventricular function. The prominent physical characteristic is the desensitization and downregulation of $\beta$-adrenergic receptors modulated by GRK2 (31). The $\beta$-adrenergic receptor regulates the normal function of the heart and the activation of myocardial $\beta$-adrenergic receptors leads to the activation of cAMP signaling pathways and increased ventricular ejection, heart rate and cardiac output. Overexpression of cardiac GRK2 leads to sympathetic hyperactivity and the attenuation of $\beta$-adrenergic receptor activity, which promotes the development of HF. To elucidate the role of GRK2 in the development of HF, lymphocyte GRK2 may be a surrogate for myocardial GRK2 in human heart disease (32). Evidence suggests that myocardial GRK 2 activity is increased almost three fold in the ventricles of patients with congestive HF and the protein and mRNA levels of GRK2 are also increased (33). With the exacerbation of hemodynamic dysfunction and HF, the expression of lymphocyte GRK2 is also upregulated in HF (34). Thus, adrenal GRK2 appears to be a major regulator of the sympathetic stimulation of the heart. Recently, the selective serotonin reuptake inhibitor antidepressant paroxetine 

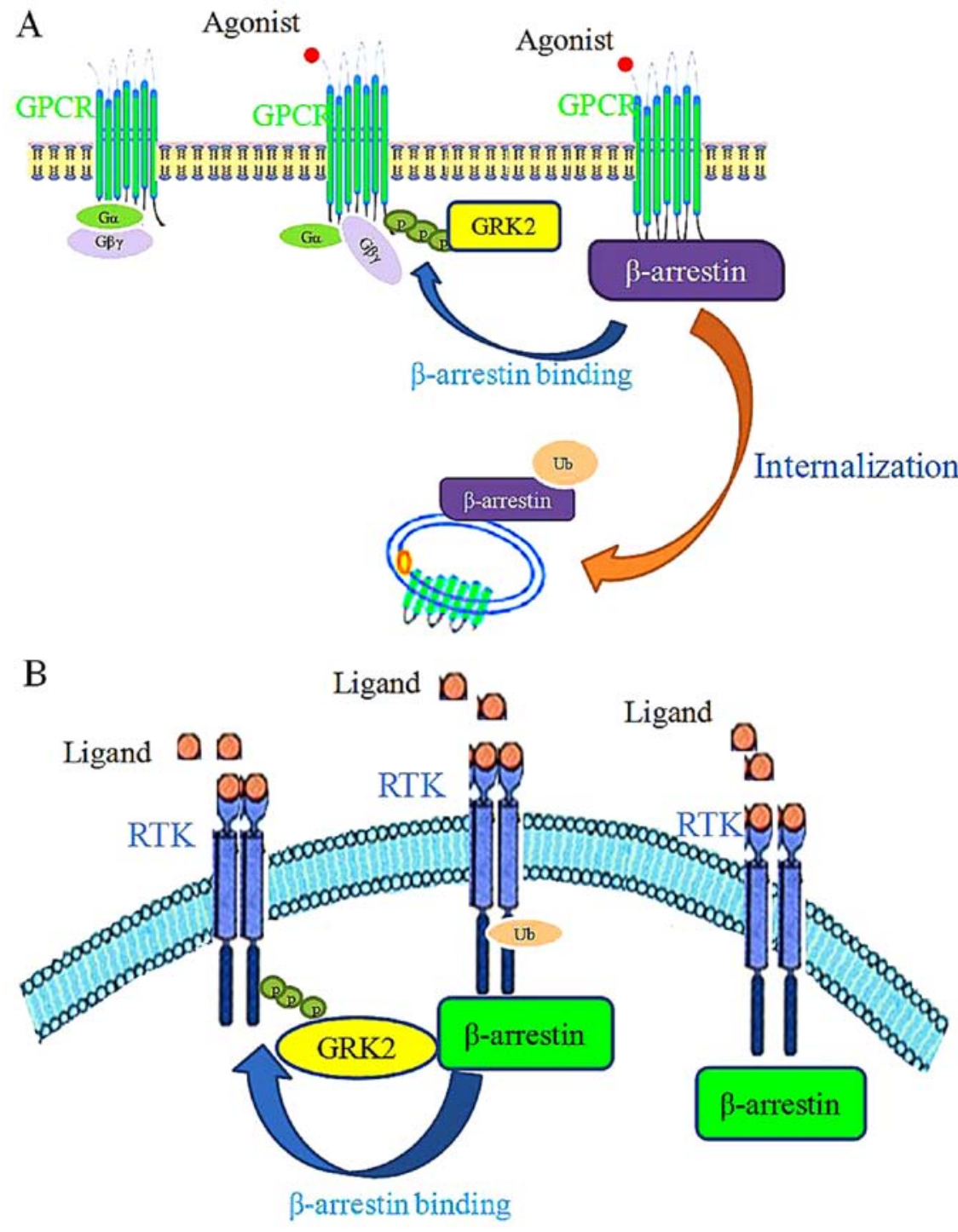

Figure 2. G protein-coupled receptor kinase 2 (GRK2) phosphorylates G protein-coupled receptors (GPCRs) and non-GPCRs. Proposed model for GRK2 regulation of GPCR and non-GPCR signaling pathways. (A) GPCR activation sequentially promotes phosphorylation by GRK2 and $\beta$-arrestin ( $\beta$-arr) binding to the phosphorylated receptor, which uncouples the receptors from $\mathrm{G}$ proteins, and target the receptors for desensitization and internalization. (B) Receptor tyrosine kinase (RTK) activation promotes phosphorylation by GRK2 binding to the phosphorylated receptor, and G protein is not involved in the phosphorylation of GRK2 on non-GPCRs, and the association of $\beta$-arrl and insulin-like growth factor-1 receptor (IGF-1R) is one of transient binding

was shown to play a role as a selective inhibitor of GRK2 activity both in vitro and in living cells (35). It specifically bound to the catalytic domain of GRK2 as an off-target and inhibited kinase activity in the micromolar range of affinity and inhibited the phosphorylation of the thyrotropin-releasing hormone receptor by GRK2 (36). In a mouse model of HF, paroxetine-mediated inhibition of GRK2 may have improves cardiovascular signaling and function (37). Chronic paroxetine treatment improves cardiac function, reverses sympathetic nervous system overdrive and normalizes the myocardial $\beta$-adrenergic system after myocardial infarction.

Potential role of GRK2 in hypertension. The exploration of GRK2 functions provided a novel research area for developing treatments for hypertension. Essential hypertension is the most dangerous factor in the development of myocardial infarction, HF and cerebrovascular disease. GRK2 plays an important role in the development of hypertension (25).
Moreover, GRK2 is also involved in the cell cycle as well as the migration and differentiation of vascular smooth muscle. GRK2 protein levels and activity in peripheral blood lymphocytes are also increased in hypertensive patients (38). With the increased GRK2-mediated phosphorylation of $\beta$-adrenergic receptor, the diastolic function of blood vessels weakens and blood pressure increases. GRK2 overexpression in vascular smooth muscle cells in mice decreased the $\beta$-adrenergic receptor signal transduction and leds to myocardial hypertrophy and hemal wall thickening (39). Based on previous research, the increased GRK2 expression in the arterial smooth muscle may enhance the proliferation and migration of vascular smooth muscle cells leading to atherosclerosis (40).

Potential role of GRK2 in inflammatory and immune diseases. Rheumatoid arthritis (RA) is a chronic, destructive autoimmune and inflammatory disease characterized by chronic synovial 
inflammation and progressive articular damage to multiple joints (41). FLSs, the key effector cells in the inflamed joint, release many proinflammatory and matrix-degrading effector molecules (42). Alterations in GRK2 levels in immune cells play an important role in the development of inflammation. A previous study indicated that a chronic inflammatory process in CIA induces the upregulation of GRK2 expression in FLSs (43). Paeoniflorin is a monoterpene glucoside and the main component of the total glucosides of paeony (TGP) extracted from the roots of Paeonia lactiflora (Paeoniaceae), which has been reported to be therapeutically effective in the treatment of RA, to decrease GRK2 expression in FLSs in vitro and to suppress the proliferation of FLSs in CIA. Downregulation of GRK2 may be one of the most important mechanisms through which Pae suppresses the proliferation of FLSs in CIA.

Potential role of GRK2 in Alzheimer's disease (AD). AD is a neurodegenerative disorder, which leads to the loss of memory and other cognitive functions (44). GRK2 is an important protein in a unifying theory of AD pathogenesis, and the change in GRK2 expression is an early contributor to the development of AD pathology. The mRNA and protein expression of GRK2 was upregulated in the lymphocytes of AD patients compared with the controls (45) as well as in post-mortem hippocampal tissues in AD patients and in rats with chronic brain hypoperfusion (46). Mounting evidence points to the possible involvement of GRK2 in the early pathogenesis of AD. Overexpression of GRK2 is a primary hallmark of mitochondrial lesions during early AD, and subcellular localization of GRK2 immunoreactivity demonstrated that GRK2 immunoreactivity was associated with damaged cellular compartments, particularly mitochondria and/or mitochondria-derived lysosomes or granular/vacuolar degenerative structures (47). Therefore, the downregulated expression of GRK2 may become a novel therapeutic strategy for AD.

Potential role of GRK2 in cancer. The finding that GRK2 regulates chemokine receptors, thyroid stimulating hormone receptor and other members of the GPCR family, has opened new fields of research into the mechanism of tumor development and into the exploitation of targeted drugs. It has been demonstrated that overexpression of GRK2 inhibits the growth of human hepatocellular carcinoma cells (48). Compared with the transfection of GRK2 kinase-defective mutant K220R in liver cancer cells, the transfection of GRK2 wt markedly decreased the proliferation of liver cancer cells. Further research showed that GRK2 overexpression induces the phosphorylation of p53 in liver cancer cells, which inhibits liver cancer cell growth. Thus, GRK2 overexpression may provide a novel perspective for the treatment of liver cancer.

\section{Conclusion}

GRK2, a typical GPCR kinase, promotes the phosphorylation and internalization of GPCRs and some non-GPCRs. The study of the structure and function of GRK2 indicated that GRK2 is composed of 689 amino acid residues and three important domain structures: an N-terminal domain, catalytic domains and a C-terminal domain (49). Each domain structure has a unique function. The composition of the N-terminal domain of approxiamtely 185 amino acids allows it to combine with $\mathrm{G} \alpha / 11$, $\mathrm{G} \beta \gamma, \alpha$-actin, caveolin and calmodulin for example, and contains PKC and c-Src phosphorylation sites. The catalytic domain is composed of approximately 270 amino acids and includes an S-nitrosylation site (Cys340), and an important locus for determining GRK2 catalytic activity (Lys220). The composition of the C-terminal domain of approximately 230 amino acids allows it to combine with PI3K, Akt, PIP2, clathrin, G $\beta \gamma$, caveolin and calmodulin, and includes PKA and ERK phosphorylation sites.

GRK2 is highly expressed in the brain, leukocytes, heart and spleen, and this expression pattern suggests that GRK2 plays an important role in neurotransmission, cardiovascular function and immune and inflammatory responses. Previous studies showed that the expression of GRK2 in peripheral blood lymphocytes was increased in HF. GRK2 levels may reflect haemodynamic impairment and may be of prognostic value following myocardial infarction. GRK2 protein levels and activity in peripheral blood lymphocytes are increased in hypertensive patients, which suggest that upregulated GRK2 levels may induce metabolic alterations and lead to insulin resistance, a common feature observed in hypertensive patients. A chronic inflammatory process in CIA induces the upregulation of GRK2 expression in FLSs, which may be one of the important mechanisms responsible for the change in the expression of GRK2 and in the proliferation of FLSs.

The antidepressant paroxetine and the stabilization of off-pathway conformational states unique to GRK2 offer a direction for the development of GRK2 inhibitors and even more selective inhibitors. Previous findings showed that paroxetine as an inhibitor of GRK2 exhibits 50-fold selectivity over other GRKs; the binding of paroxetine to the active site of the GRK2 kinase domain is very high, and the benzodioxole ring of paroxetine interacts with the hinge of GRK2. Paroxetine, a selective GRK2 inhibitor, has applications in the reversal of cardiac dysfunction and remodeling after myocardial infarction. The vast amount of research regarding GRK2 inhibitors suggest that the potential therapeutic effects of GRK2 inhibition may lead to novel candidates for the prevention and treatment of HF, hypertension and inflammatory diseases. The application of emerging technologies as well as gene knockout studies, gene cloning and the progress of research into functional genomics and proteomics will enable studies of GRK2 to achieve breakthroughs.

\section{Acknowledgements}

This study was supported by the National Natural Science Foundation of China (nos. 81502123 and 81330081), the Natural Science Foundation of Anhui Province (no. 1308085QH130), and the Anhui Province Nature Science Foundation in University (no. KJ2014A119).

\section{References}

1. Evron T, Daigle TL, Caron MG, Rivas V, Nogués L, Lucas E, Vila-Bedmar R, Murga C and Mayor F Jr: GRK2: multiple roles beyond $\mathrm{G}$ protein-coupled receptor desensitization. Trends Pharmacol Sci 33: 154-164, 2012.

2. Penela P, Murga C, Ribas C, Lafarga V and Mayor F Jr: The complex G protein-coupled receptor kinase 2 (GRK2) interactome unveils new physiopathological targets. Br J Pharmacol 160: 821-832, 2010. 
3. Cannavo A, Liccardo D and Koch WJ: Targeting cardiac $\beta$-adrenergic signaling via GRK2 inhibition for heart failure therapy. Front Physiol 4: 264, 2013.

4. Piao L, Fang YH, Parikh KS, Ryan JJ, D'Souza KM, Theccanat T, Toth PT, Pogoriler J, Paul J, Blaxall BC, et al: GRK2-mediated inhibition of adrenergic and dopaminergic signaling in right ventricular hypertrophy: therapeutic implications in pulmonary hypertension. Circulation 126: 2859-2869, 2012

5. Elorza A, Sarnago S and Mayor F Jr: Agonist-dependent modulation of $\mathrm{G}$ protein-coupled receptor kinase 2 by mitogenactivated protein kinases. Mol Pharmacol 57: 778-783, 2000.

6. Whalen EJ, Foster MW, Matsumoto A, Ozawa K, Violin JD, Que LG, Nelson CD, Benhar M, Keys JR, Rockman HA, et al: Regulation of $\beta$-adrenergic receptor signaling by $S$-nitrosylation of G-protein-coupled receptor kinase 2. Cell 129: 511-522, 2007.

7. Eichmann T, Lorenz K, Hoffmann M, Brockmann J, Krasel C, Lohse MJ and Quitterer U: The amino-terminal domain of G-protein-coupled receptor kinase 2 is a regulatory Gbeta gamma binding site. J Biol Chem 278: 8052-8057, 2003.

8. Houslay MD and Baillie GS: Phosphodiesterase-4 gates the ability of protein kinase A to phosphorylate G-protein receptor kinase-2 and influences its translocation. Biochem Soc Trans 34: 474-475, 2006

9. Granier S, Kim S, Shafer AM, Ratnala VR, Fung JJ, Zare RN and Kobilka B: Structure and conformational changes in the C-terminal domain of the $\beta_{2}$-adrenoceptor: insights from fluorescence resonance energy transfer studies. J Biol Chem 282: 13895-13905, 2007.

10. Reiter E and Lefkowitz RJ: GRKs and $\beta$-arrestins: roles in receptor silencing, trafficking and signaling. Trends Endocrinol Metab 17: 159-165, 2006.

11. Nobles KN, Xiao K, Ahn S, Shukla AK, Lam CM, Rajagopal S, Strachan RT, Huang TY, Bressler EA, Hara MR, et al: Distinct phosphorylation sites on the $\beta(2)$-adrenergic receptor establish a barcode that encodes differential functions of $\beta$-arrestin. Sci Signal 4: ra51, 2011.

12. de Gasparo M, Catt KJ, Inagami T, Wright JW and Unger T: International union of pharmacology. XXIII. The angiotensin II receptors. Pharmacol Rev 52: 415-472, 2000.

13. Violin JD, Dewire SM, Barnes WG and Lefkowitz RJ $\mathrm{G}$ protein-coupled receptor kinase and beta-arrestin-mediated desensitization of the angiotensin II type $1 \mathrm{~A}$ receptor elucidated by diacylglycerol dynamics. J Biol Chem 281: 36411-36419, 2006.

14. Rajagopal K, Whalen EJ, Violin JD, Stiber JA, Rosenberg PB, Premont RT, Coffman TM, Rockman HA and Lefkowitz RJ: Beta-arrestin2-mediated inotropic effects of the angiotensin II type $1 \mathrm{~A}$ receptor in isolated cardiac myocytes. Proc Natl Acad Sci USA 103: 16284-16289, 2006

15. Kleibeuker W, Jurado-Pueyo M, Murga C, Eijkelkamp N, Mayor F Jr, Heijnen CJ and Kavelaars A: Physiological changes in GRK2 regulate CCL2-induced signaling to ERK1/2 and Akt but not to MEK1/2 and calcium. J Neurochem 104: 979-992, 2008.

16. Lipfert J, Ödemis V and Engele J: Grk2 is an essential regulator of CXCR7 signalling in astrocytes. Cell Mol Neurobiol 33: 111-118, 2013.

17. Neuschäfer-Rube F, Oppermann M, Möller U, Böer U and Püschel GP: Agonist-induced phosphorylation by G proteincoupled receptor kinases of the EP4 receptor carboxyl-terminal domain in an EP3/EP4 prostaglandin E(2) receptor hybrid. Mol Pharmacol 56: 419-428, 1999.

18. Wang Y, Gao E, Lau WB, Wang Y, Liu G, Li JJ, Wang X, Yuan Y, Koch WJ and Ma XL: G-protein-coupled receptor kinase 2-mediated desensitization of adiponectin receptor 1 in failing heart. Circulation 131: 1392-1404, 2015

19. Kelley-Hickie LP and Kinsella BT: Homologous desensitization of signalling by the beta (beta) isoform of the human thromboxane A2 receptor. Biochim Biophys Acta 1761: 1114-1131, 2006

20. Kanaide M, Uezono Y, Matsumoto M, Hojo M, Ando Y, Sudo Y, Sumikawa K and Taniyama K: Desensitization of GABA(B) receptor signaling by formation of protein complexes of GABA(B2) subunit with GRK4 or GRK5. J Cell Physiol 210: 237-245, 2007.

21. Terunuma M,Pangalos MN and Moss SJ: Functional modulation of GABAB receptors by protein kinases and receptor trafficking. Adv Pharmacol 58: 113-122, 2010

22. Iacovelli L, Capobianco L, D'Ancona GM, Picascia A and De Blasi A: Regulation of lysophosphatidic acid receptorstimulated response by G-protein-coupled receptor kinase-2 and beta-arrestin1 in FRTL-5 rat thyroid cells. J Endocrinol 174: $103-110,2002$
23. Cho DI, Zheng M, Min C, Kwon KJ, Shin CY, Choi HK and Kim KM: ARF6 and GASP-1 are post-endocytic sorting proteins selectively involved in the intracellular trafficking of dopamine $\mathrm{D}_{2}$ receptors mediated by GRK and PKC in transfected cells. $\mathrm{Br}$ J Pharmacol 168: 1355-1374, 2013.

24. Namkung Y, Dipace C, Urizar E, Javitch JA and Sibley DR: $G$ protein-coupled receptor kinase-2 constitutively regulates $D_{2}$ dopamine receptor expression and signaling independently of receptor phosphorylation. J Biol Chem 284: 34103-34115, 2009.

25. Chen Y, Sasai N, Ma G, Yue T, Jia J, Briscoe J and Jiang J: Sonic Hedgehog dependent phosphorylation by CK1 $\alpha$ and GRK2 is required for ciliary accumulation and activation of smoothened. PLoS Biol 9: e1001083, 2011.

26. Evron T, Philipp M, Lu J, Meloni AR, Burkhalter M, Chen W and Caron MG: Growth Arrest Specific 8 (Gas8) and G protein-coupled receptor kinase 2 (GRK2) cooperate in the control of Smoothened signaling. J Biol Chem 286: 27676-27686, 2011.

27. Peppel K, Jacobson A, Huang X, Murray JP, Oppermann M and Freedman NJ: Overexpression of $G$ protein-coupled receptor kinase-2 in smooth muscle cells attenuates mitogenic signaling via $\mathrm{G}$ protein-coupled and platelet-derived growth factor receptors. Circulation 102: 793-799, 2000.

28. Wei Z, Hurtt R, Gu T, Bodzin AS, Koch WJ and Doria C: GRK2 negatively regulates IGF-1R signaling pathway and cyclins expression in HepG2 cells. J Cell Physiol 228: 1897-1901, 2013.

29. Zheng H, Worrall C, Shen H, Issad T, Seregard S, Girnita A and Girnita L: Selective recruitment of $G$ protein-coupled receptor kinases (GRKs) controls signaling of the insulin-like growth factor 1 receptor. Proc Natl Acad Sci USA 109: 7055-7060, 2012.

30. So CH, Michal A, Komolov KE, Luo J and Benovic JL: $\mathrm{G}$ protein-coupled receptor kinase 2 (GRK2) is localized to centrosomes and mediates epidermal growth factor-promoted centrosomal separation. Mol Biol Cell 24: 2795-2806, 2013.

31. Huang ZM, Gao E, Chuprun JK and Koch WJ: GRK2 in the heart: a GPCR kinase and beyond. Antioxid Redox Signal 21 2032-2043, 2014.

32. Rengo G, Pagano G, Paolillo S, de Lucia C, Femminella GD, Liccardo D, Cannavo A, Formisano R, Petraglia L, Komici $\mathrm{K}$, et al: Impact of diabetes mellitus on lymphocyte GRK2 protein levels in patients with heart failure. Eur J Clin Invest 45: 187-195, 2015.

33. Dorn GW II: GRK mythology: G-protein receptor kinases in cardiovascular disease. J Mol Med Berl 87: 455-463, 2009.

34. Rengo G, Perrone-Filardi P, Femminella GD, Liccardo D, Zincarelli C, de Lucia C, Pagano G, Marsico F, Lymperopoulos A and Leosco D: Targeting the $\beta$-adrenergic receptor system through G-protein-coupled receptor kinase 2: a new paradigm for therapy and prognostic evaluation in heart failure: from bench to bedside. Circ Heart Fail 5: 385-391, 2012.

35. Homan KT, Wu E, Wilson MW, Singh P, Larsen SD and Tesmer JJ: Structural and functional analysis of $g$ protein-coupled receptor kinase inhibition by paroxetine and a rationally designed analog. Mol Pharmacol 85: 237-248, 2014

36. Schumacher SM, Gao E, Zhu W, Chen X, Chuprun JK, Feldman AM, Tesmer JJ and Koch WJ: Paroxetine-mediated GRK2 inhibition reverses cardiac dysfunction and remodeling after myocardial infarction. Sci Transl Med 7: 277ra31, 2015.

37. Thal DM, Homan KT, Chen J, Wu EK, Hinkle PM, Huang ZM, Chuprun JK, Song J, Gao E, Cheung JY, et al: Paroxetine is a direct inhibitor of $\mathrm{g}$ protein-coupled receptor kinase 2 and increases myocardial contractility. ACS Chem Biol 7: 1830-1839, 2012.

38. Santulli G, Trimarco B and Iaccarino G: G-protein-coupled receptor kinase 2 and hypertension: molecular insights and pathophysiological mechanisms. High Blood Press Cardiovasc Prev 20: 5-12, 2013.

39. Eckhart AD, Ozaki T, Tevaearai H, Rockman HA and Koch WJ. Vascular-targeted overexpression of $G$ protein-coupled receptor kinase- 2 in transgenic mice attenuates beta-adrenergic receptor signaling and increases resting blood pressure. Mol Pharmacol 61: 749-758 2002

40. Erbel R, Lehmann N, Möhlenkamp S, Churzidse S, Bauer M, Kälsch H, Schmermund A, Moebus S, Stang A, Roggenbuck U, et al; Heinz Nixdorf Recall Study Investigators: Subclinical coronary atherosclerosis predicts cardiovascular risk in different stages of hypertension: result of the Heinz Nixdorf Recall Study. Hypertension 59: 44-53, 2012.

41. Bax M, van Heemst J, Huizinga TW and Toes RE: Genetics of rheumatoid arthritis: What have we learned? Immunogenetics 63: $459-466,2011$ 
42. Bartok B and Firestein GS: Fibroblast-like synoviocytes: key effector cells in rheumatoid arthritis. Immunol Rev 233: 233-255, 2010.

43. Chen JY, Wu HX, Chen Y, Zhang LL, Wang QT, Sun WY and Wei W: Paeoniflorin inhibits proliferation of fibroblast-like synoviocytes through suppressing G-protein-coupled receptor kinase 2. Planta Med 78: 665-671,2012.

44. Nelson PT, Braak H and Markesbery WR: Neuropathology and cognitive impairment in Alzheimer disease: a complex bu coherent relationship. J Neuropathol Exp Neurol 68: 1-14, 2009.

45. Obrenovich ME, Smith MA, Siedlak SL, Chen SG, de la Torre JC, Perry G and Aliev G: Overexpression of GRK2 in Alzheimer disease and in a chronic hypoperfusion rat model is an early marker of brain mitochondrial lesions. Neurotox Res 10: 43-56, 2006.
46. Obrenovich ME, Morales LA, Cobb CJ, Shenk JC, Méndez GM, Fischbach K, Smith MA, Qasimov EK, Perry G and Aliev G: Insights into cerebrovascular complications and Alzheimer disease through the selective loss of GRK2 regulation. J Cell Mol Med 13: 853-865, 2009.

47. Suo WZ and Li L: Dysfunction of G protein-coupled receptor kinases in Alzheimer's disease. ScientificWorldJournal 10: $1667-1678,2010$

48. Wei Z, Hurtt R, Ciccarelli M, Koch WJ and Doria C: Growth inhibition of human hepatocellular carcinoma cells by overexpression of G-protein-coupled receptor kinase 2. J Cell Physiol 227: 2371-2377, 2012.

49. Penela P, Ribas C and Mayor F Jr: Mechanisms of regulation of the expression and function of $\mathrm{G}$ protein-coupled receptor kinases. Cell Signal 15: 973-981, 2003. 